\title{
\#Vaccine: Using Hashtags from Indian Tweets to Capture and Analyse the Sentiments of People on Vaccination for Covid'19 Pandemic
}

\author{
K. Anuratha ${ }^{\mathrm{a}, 1}$, S.Sujeetha ${ }^{\mathrm{a}}$, J.M.Nandhini ${ }^{\mathrm{a}}$, B.Priya ${ }^{\mathrm{a}}$ and M.Paravthy ${ }^{\mathrm{b}}$ \\ ${ }^{a}$ Dept of Information Technology, Sri Sai Ram Institute of Technology, Chennai, \\ Tamilnadu, India \\ ${ }^{b}$ Department of CSE, Sethu Institute of Technology, Madurai, Tamilnadu, India
}

\begin{abstract}
To prevent the public from pandemic Covid'19 the government of India has started the vaccination from mid of January 2021. The government has approved the two vaccines, Covishield from the university of Oxford and Covaxin from Bharat Biotech.The vaccination started with frontline workers and is further extended to common public prioritizing the elders of above 60 years and people aged 45 years above with co morbidities. Though many people have got benefitted from it there is still a group of people not convinced with the vaccination. We have carried out this work to analyze those Indian people sentiments on the vaccines through the hash tags of tweets. The results show that though majority of the community has a positive belief on the vaccines but some of them still express negative emotions.
\end{abstract}

Keywords. COVID, Vaccine, India, Covaxin, Covishield, Pandemic

\section{Introduction}

The covid-19 pandemic has infected about billions of people across the globe and deaths [1]. Major countries have imposed lockdown at different phases in 2020 but it's not the feasible solution considering the economic benefits of any nation. The only possible solution is to break the chain of the infection and is possible only through immunizing the public with vaccination. As India is one among the country with majority of population affected by the pandemic, government has announced the vaccination from January 2021. The nation has approved two vaccines covishield and covaxin from the University of Oxford and Bharat Biotech respectively.

\footnotetext{
${ }^{1}$ K. Anuratha, Assistant Professor, Department of IT, Sri Sai Ram Institute of Technology, Chennai Email: anuvinaya2003@gmail.com.
} 
The vaccination benefits for about 40 million of healthcare workers and frontline workers. The nation aims at benefitting for a about 300 million people on priority basis [2]. As the Government of India has approved the two vaccines as mentioned and also there is huge demand and difficulties in logistics it's not possible for the individuals to opt for the vaccine. They need to proceed with the plans of the government [3]. The people, who are vaccinated and waiting for their chance to get vaccinated, express their views in social platforms. In this work we made use of emotion-word hash tags used in twitter in analyzing the views of general public towards the government initiative to contain the pandemic in India. Twitter has become the popular medium for day to today information sharing on various fields. It has around 400 billion active users, daily. Not only for information sharing, public uses this platform for sharing their thoughts. Researchers make use of these opinions to analyze the sentiments of peoples on various issues like politics, sports, product reviews etc., The emotions expressed by the Twitter community during 2020 has been used to understand the people sentiments during a pandemic [7-12].

\section{Literature Review}

In [13] the authors have used the Twitter data with the covid-19 keyword to analyses the sentiments of Indonesian public towards the vaccine for covid. They have shown negative sentiments were more than that of positive sentiments. Deep learning algorithms have become popular in recent years for sentiment analysis [14].

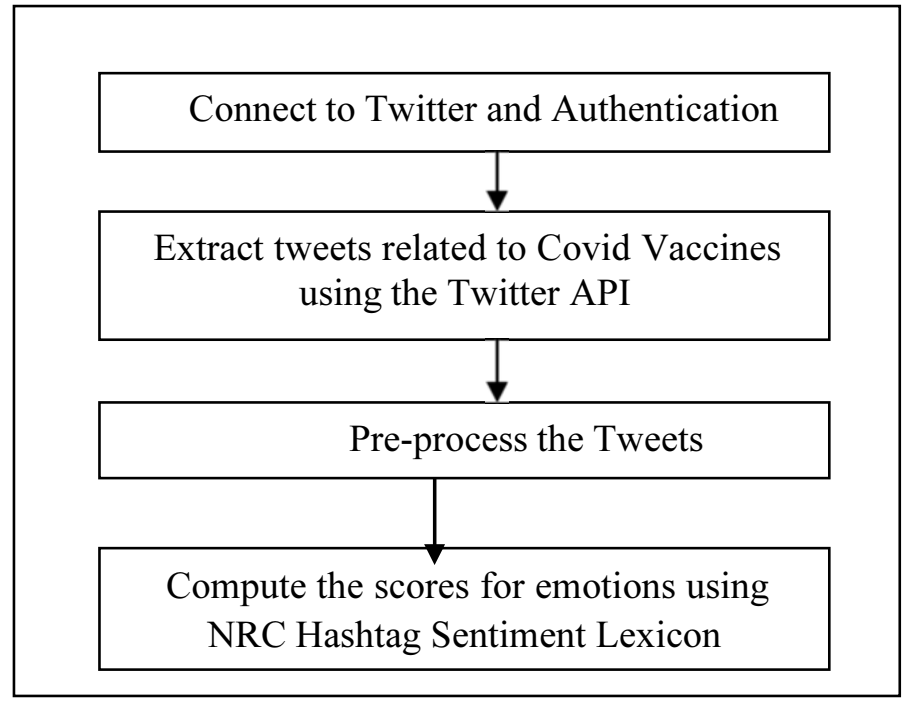

Figure 1. Steps in analysing the sentiments expressed in tweets 


\section{Proposed Methodology}

The emotions expressed in the tweets extracted using the hashtags (\#) are analyzed using deep learning algorithms as mentioned in the figure 1

As we have to analyse the Indian tweets associated to vaccine covishield and covaxin we have prepared two different data sets with the tweets collected during January and February 2021. Once we are done with the data sets, we have pre-processed the data. Then we have adopted the NRC hash tag sentiment lexicon to categorize the tweets in to emotions anticipation, joy, anger, disgust, fear, trust and surprise.

\section{Results and Discussion}

The results of the research are discussed here. Figure 2 shows people have tweeted with $82 \%$ positive sentiments and $18 \%$ negative sentiments for the vaccine covishield. For Covaxin, the tweets with positive sentiments were with $68 \%$ and $32 \%$ were with negative sentiments.

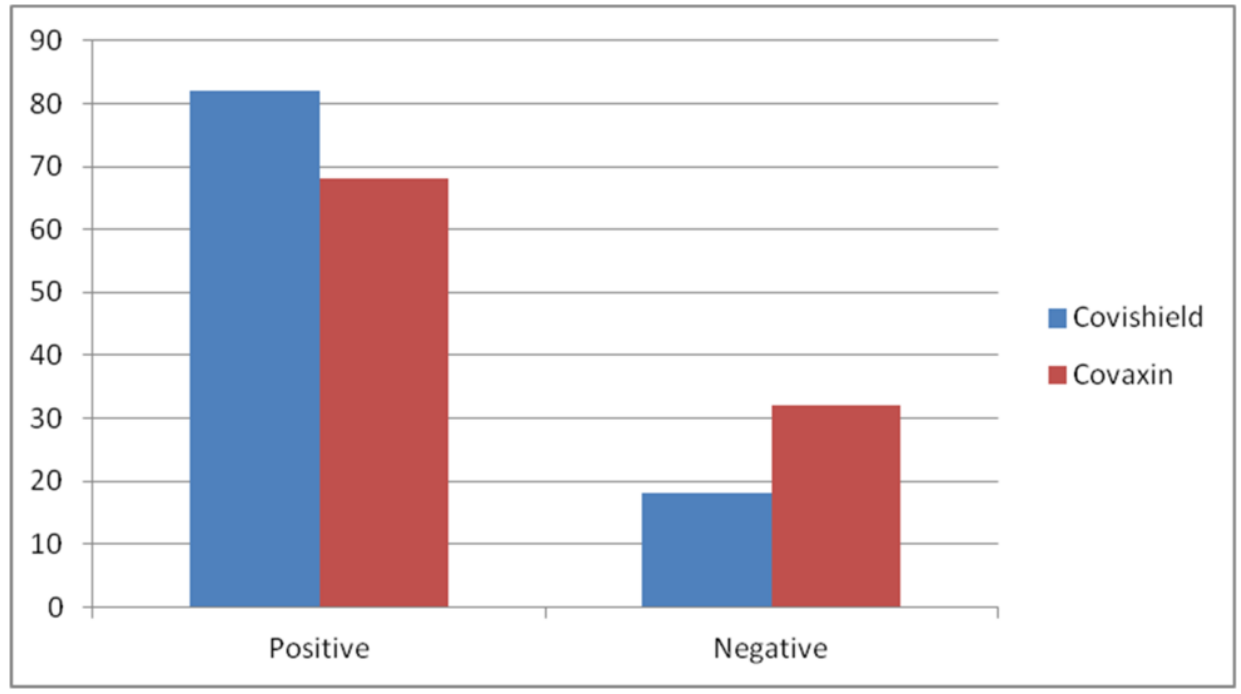

Figure 2. Sentiment Analysis of Indian Tweets on the Vaccines for Covid'19

Then, the emotions on the tweets were analyzed and the same is mentioned in Figure 3. It is observed that the emotions joy, trust, anticipation on Covishield is high than that of Covaxin. The emotions anger, fear and digest associated with Covaxin is high than Covishield trust and anticipation emotions associated with Covishield was more than the value of trust and anticipation associated with Covaxin. While the emotions surprise and sadness associated with both are equal. 


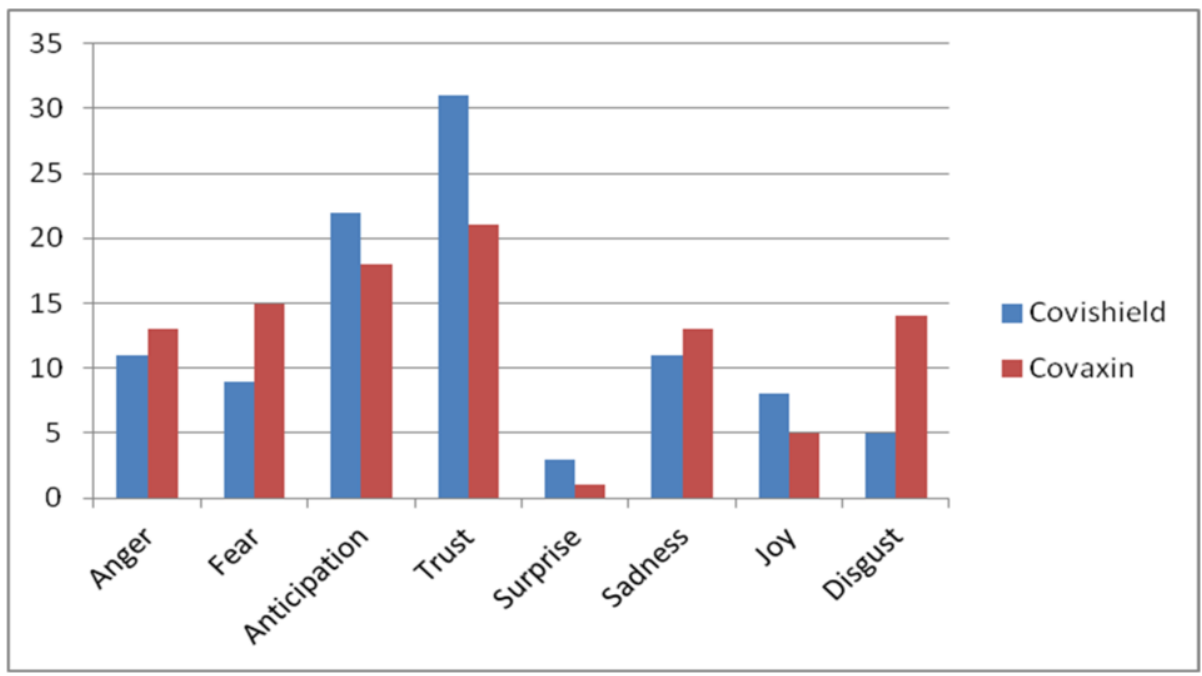

Figure 3. Emotion Analysis of Indian Tweets on the Vaccines for Covid'19

\section{Conclusion}

This work was carried out to analyze the impact of Vaccination in India through tweets of people. It was observed that the vaccine Covishield had positive sentiments higher than that of Covaxin. Also, the emotions like trust, anticipation. In the study, it was found that the tweets regarding Covishield had more positive sentiments as compared to Covaxin. Emotions like trust and anticipation were more in tweets associated to Covishield. The emotions anger and disgust were expressed more for tweets associated to Covaxin as compared to Covishield.

\section{References}

[1] Jagdale, Rajkumar S., Vishal S. Shirsat, and Sachin N. Deshmukh. "Sentiment analysis on product reviews using machine learning techniques." In Cognitive Informatics and Soft Computing, pp. 639- 647. Springer, Singapore, 2019.

[2] UMA RAMYA, V; THIRUPATHI RAO, K. Sentiment Analysis of Movie Review using Machine Learning Techniques. International Journal of Engineering \& Technology, [S.1.], v. 7, n. 2.7, p. 676-681, mar. 2018.

[3] M A, Ravikumar P. Survey: Twitter data Analysis using Opinion Mining. International Journal of Computer Applications. 2015;128(5):34-36.

[4] UPI Systems in India Post Demonetization Using IBM Watson." In 2018 International Conference on Computer Communication and Informatics (ICCCI), pp. 1-6. IEEE, 2018.

[5] Wang Y, Taylor J. Coupling sentiment and human mobility in natural disasters: a Twitter-based study of the 2014 South Napa Earthquake. Natural Hazards. 2018;92(2):907-925.

[6] Dubey, A. D. (2020). Twitter sentiment analysis during COVID19 outbreak. Available at SSRN 3572023.

[7] Manguri, K. H., Ramadhan, R. N., \& Amin, P. R. M. (2020). Twitter sentiment analysis on worldwide COVID-19 outbreaks. Kurdistan Journal of Applied Research, 54-65.

[8] Bhat, M., Qadri, M., Noor-ul-Asrar Beg, M. K., Ahanger, N., \& Agarwal, B. (2020). Sentiment analysis of social media response on the covid19 outbreak. Brain, Behavior, and Immunity.

[9] Pastor, C. K. (2020). Sentiment analysis of Filipinos and effects of extreme community quarantine due to coronavirus (Covid-19) pandemic. Available at SSRN 3574385 
[10] Pristiyono et al 2021 IOP Conf. Ser.: Mater. Sci. Eng. 1088012045

[11] Rev., Data Mining Knowl. Discovery, vol. 8, no. 4, Jul. 2018, Art. no. e1253, doi: 10.1002/widm. 1253 . 